\title{
New Generating Function Relations for the $q$-Generalized Cesàro Polynomials
}

\author{
Nejla Özmen $\mathbb{D}$ \\ Düzce University, Faculty of Art and Science, Department of Mathematics, Konuralp 81620, Düzce, Turkey \\ Correspondence should be addressed to Nejla Özmen; nejlaozmen06@gmail.com
}

Received 27 February 2019; Accepted 25 March 2019; Published 24 April 2019

Guest Editor: Tuncer Acar

Copyright (C) 2019 Nejla Özmen. This is an open access article distributed under the Creative Commons Attribution License, which permits unrestricted use, distribution, and reproduction in any medium, provided the original work is properly cited.

The main purpose of this paper is to examine a basic (or q-) analogue of the generalized Cesàro polynomials described here. We derive a bilateral $q$-generating function involving basic analogue of Fox's $H$-function and $q$-generalized Cesàro polynomials.

\section{Introduction}

The Cesàro polynomials $g_{n}^{(s)}(x)$ are defined by the generating relation ([1], p. 449, Problem 20):

$$
\sum_{n=0}^{\infty} g_{n}^{(s)}(x) t^{n}=(1-t)^{-s-1}(1-x t)^{-1}
$$

It is from (1) that

$$
g_{n}^{(s)}(x)=\left(\begin{array}{c}
s+n \\
n
\end{array}\right){ }_{2} F_{1}[-n, 1 ;-s-n ; x]
$$

where ${ }_{2} F_{1}$ denotes Gauss's hypergeometric series.

Lin et al. [2] introduced the generalized Cesàro polynomials as follows:

$$
g_{n}^{(s)}(\lambda, x)=\left(\begin{array}{c}
s+n \\
n
\end{array}\right){ }_{2} F_{1}[-n, \lambda ;-s-n ; x]
$$

It is noted that the special case $\lambda=1$ of (3) reduces immediately to the Cesàro polynomials defined by (2).
Furthermore, they satisfy the generating functions [3]:

$$
\sum_{n=0}^{\infty} g_{n}^{(s)}(\lambda, x) t^{n}=(1-t)^{-s-1}(1-x t)^{-\lambda}
$$

and

$$
\begin{aligned}
& \sum_{n=0}^{\infty}\left(\begin{array}{c}
n+m \\
n
\end{array}\right) g_{n+m}^{(s)}(\lambda, x) t^{n} \\
& \quad=(1-t)^{-s-m-1}(1-x t)^{-\lambda} g_{m}^{(s)}\left(\lambda, \frac{x(1-t)}{1-x t}\right),
\end{aligned}
$$

where $m=0,1,2, \ldots$.

The purpose of this study is to obtain $q$-analogue of generalized Cesàro polynomials as $q$-analogue of the production functions mentioned above. The structure of this paper is as follows.

In Section 2, we give some preliminaries on $q$-calculus. In Section 3, we define some $q$-analogue of Cesàro polynomials. In Section 4, theorems are given for bilinear and bilateral generating functions for $q$-generalized Cesàro polynomials. In Section 5, the application of the theorems given in Section 4 will be given.

\section{Some $q$-Calculus: The Definitions}

Let $q \in \mathbb{C}, 0<|q|<1$. A $q$-analogue of the hypergeometric series ${ }_{p} F_{r}$ is the basic hypergeometric series [4]: 


$$
\begin{aligned}
& { }_{r} \phi_{s}\left[\begin{array}{l}
\alpha_{1}, \ldots, \alpha_{r} \\
\beta_{1}, \ldots, \beta_{s}
\end{array}\right] \\
& =\sum_{k=0}^{\infty} \frac{\left(\alpha_{1} ; q\right)_{k} \cdots\left(\alpha_{r} ; q\right)_{k}}{\left(\beta_{1} ; q\right)_{k} \cdots\left(\beta_{s} ; q\right)_{k}}\left((-1)^{k} q^{k(k-1) / 2}\right)^{1+r+s} \frac{z^{k}}{(q ; q)_{k}},
\end{aligned}
$$

where $q \neq 0$ when $r>s+1$, and $\left(\beta_{i}\right)$ are such that the denominator never vanishes. We also need to define some other $q$-analogues, such as the $q$-analogue of a number $[\alpha]_{q}$, factorial $[\alpha]_{q}$ !, and the Pochhammer symbol (rising factorial) $(\alpha)_{n}$. These $q$-analogues are given as follows:

$$
\begin{aligned}
{[\alpha]_{q} } & =\frac{1-q^{\alpha}}{1-q}, \\
{[0]_{q} ! } & =1, \\
{[n]_{q} ! } & =\prod_{k=1}^{n}[k]_{q}, \quad n \in \mathbb{N} .
\end{aligned}
$$

The number $(\mu ; q)_{\omega}$ is given by

$$
(\mu ; q)_{\omega}:=\frac{(\mu ; q)_{\infty}}{\left(\mu q^{\omega} ; q\right)_{\infty}}
$$

where

$$
(\mu ; q)_{\infty}:=\prod_{s=0}^{\infty}\left(1-\mu q^{s}\right)
$$

and $\mu, \omega$ are arbitrary parameters so that

$$
\begin{aligned}
& (\mu ; q)_{\omega} \\
& := \begin{cases}1 & \text { if } \omega=0 \\
(1-\mu)(1-\mu q) \cdots\left(1-\mu q^{\omega-1}\right) & \text { if } \omega=1,2,3, \ldots ;\end{cases}
\end{aligned}
$$

see, for instance, [5], pp. 413-414.

The $q$-gamma function [4] is defined by

$$
\begin{aligned}
\Gamma_{q}(x)=\frac{(q ; q)_{\infty}}{\left(q^{x} ; q\right)_{\infty}}(1-q)^{1-x} & \\
x & \in \mathbb{C}, x \notin\{0,-1,-2, \ldots\} .
\end{aligned}
$$

And it satisfies

$$
\Gamma_{q}(x+1)=\frac{1-q^{x}}{1-q} \Gamma_{q}(x)
$$

Definition 1. The $q$-analogue of Cesàro's polynomial is defined as follows [6]:

$$
g_{n}^{(s)}(x ; q)=\frac{\left(q^{1+s} ; q\right)_{n}}{(q ; q)_{n}}{ }_{2} \phi_{1}\left[\begin{array}{c}
q^{-n}, q \\
q^{-s-n}
\end{array} ; q, x\right]=\sum_{k=0}^{n}\left[\begin{array}{c}
k+s \\
s
\end{array}\right]_{q}\left(x q^{s}\right)^{n-k},
$$

where ${ }_{2} \phi_{1}$ denotes $q$-hypergeometric function and defined by [6]

$$
{ }_{2} \phi_{1}\left[\begin{array}{c}
q^{-n}, q \\
q^{-s-n}
\end{array} q, x\right]=\sum_{k=0}^{n} \frac{\left(q^{-n} ; q\right)_{k}(q ; q)_{k}}{\left(q^{-s-n} ; q\right)_{k}} \frac{x^{k}}{(q ; q)_{k}} .
$$

Definition 2. The $q$-Cesàro polynomials satisfy the following generating function $[6,7]$ :

$$
\sum_{n=0}^{\infty} g_{n}^{(s)}(x ; q) t^{n}=\frac{1}{\left(1-q^{s} x t\right)(t ; q)_{s+1}} .
$$

Following Saxena, Modi, and Kalla [8], the basic analogue of the Fox's $H$-function is defined as

$$
H_{P, Q}^{M, N}\left[\begin{array}{cc} 
& \\
x ; q \mid & \\
& (b, \beta)
\end{array}\right]=\frac{1}{2 \pi i} \int_{C} \Phi(s ; q) x^{s} d_{q} s,
$$

where

$$
\begin{aligned}
& \Phi(s ; q) \\
& =\frac{\left\{\prod_{j=1}^{M} G\left(q^{b_{j}-\beta_{j} s}\right)\right\}\left\{\prod_{j=1}^{N} G\left(q^{1-a_{j}+\alpha_{j} s}\right)\right\} \pi}{\left\{\prod_{j=M+1}^{Q} G\left(q^{1-b_{j}+\beta_{j} s}\right)\right\}\left\{\prod_{j=N+1}^{P} G\left(q^{a_{j}-\alpha_{j} s}\right)\right\} G\left(q^{1-s}\right) \sin \pi s}
\end{aligned}
$$

and

$$
G\left(q^{s}\right)=\left\{\prod_{n=0}^{\infty}\left(1-q^{a+n}\right)\right\}^{-1}=\frac{1}{\left(q^{a} ; q\right)_{\infty}}
$$

Also $0 \leq M \leq Q, 0 \leq N \leq P, \alpha_{i}$ 's and $\beta_{j}$ 's are positive integers. The contour $C$ is a line parallel to $\operatorname{Re}(w s)=0$ with indentations if necessary, in such a manner that all the poles of $G\left(q^{b_{j}-\beta_{j} s}\right), 1 \leq j \leq M$ are to the right and those of $G\left(q^{1-a_{j}+\alpha_{j} s}\right), 1 \leq j \leq N$ are to the left of $C$. For large values of $|s|$, the integral converges if $\operatorname{Re}[s \log (x)-\log \sin \pi s]<0$ on the contour $C$, i.e., if $\left|\left\{\arg (x)-w_{2} w_{1}^{-1} \log |x|\right\}\right|<\pi$, where $0<|q|<1, \log q=-w=-\left(w_{1}+i w_{2}\right), w_{1}$ and $w_{2}$ are real.

Further, if we set $\alpha_{i}=\beta_{j}=1, \forall i$ and $j$ in (16), we obtain the basic analogue of Meijer's G-function due to Saxena, Modi, and Kalla [8]:

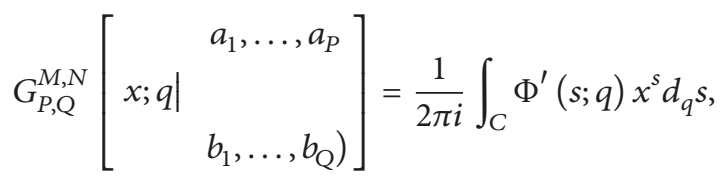

where

$$
\begin{aligned}
& \Phi^{\prime}(s ; q) \\
& =\frac{\left\{\prod_{j=1}^{M} G\left(q^{b_{j}-s}\right)\right\}\left\{\prod_{j=1}^{N} G\left(q^{1-a_{j}+s}\right)\right\} \pi}{\left\{\prod_{j=M+1}^{Q} G\left(q^{1-b_{j}+s}\right)\right\}\left\{\prod_{j=N+1}^{P} G\left(q^{a_{j}-s}\right)\right\} G\left(q^{1-s}\right) \sin \pi s} .
\end{aligned}
$$

Detailed account of Meijer's G-function, Fox's $H$-function, and various functions expressed by Fox's $H$-function can be found in the research monographs of 
Mathai and Saxena [9, 10], Srivastava, Gupta, and Goyal [11], and Mathai, Saxena, and Haubold [12]. In addition, the basic functions of a variable that can be expressed in terms of $G q(\cdot)$ functions can be found in the works of Yadav and Purohit $[13,14]$. In the last quarter of the twentieth century, the quantum calculus (also known as $q$-calculus) can be found on the theory of approaches of operators $[15,16]$.

\section{Construction of the $q-$ Generalized Cesàro Polynomials}

In this section, with the help of the similar method as considered in $[2,5,17,18]$, we form the analogue of $q$-generalized Cesàro polynomials $g_{n}^{(s)}(\lambda, x ; q)$ given by (3).

Definition 3. The $q$-generalized Cesàro polynomials $g_{n}^{(s)}(\lambda, x ; q)$ given by (3) are written as follows:

$$
\begin{aligned}
g_{n}^{(s)}(\lambda, x ; q & \left.:=\frac{\left(q^{s+1} ; q\right)_{n}}{(q ; q)_{n}}{ }_{2} \phi_{1}\left[\begin{array}{c}
q^{-n}, q^{\lambda} \\
q^{-s-n}
\end{array}\right], x\right] \\
& =\sum_{k=0}^{n}\left[\begin{array}{c}
n-k+s \\
n-k
\end{array}\right]_{q}\left(q^{\lambda} ; q\right)_{k} \frac{\left(x q^{s}\right)^{k}}{(q ; q)_{k}} .
\end{aligned}
$$

It is noted that the special case $\lambda=1$ of (21) reduces immediately to the generalized Cesàro polynomials defined by (4).

Theorem 4. The q-generalized Cesàro polynomials have the following generating function relation:

$$
\frac{1}{\left(q^{s} x t ; q\right)_{\lambda}(t ; q)_{s+1}}=\sum_{k=0}^{\infty} g_{n}^{(s)}(\lambda, x ; q) t^{n},
$$

where $|t|<\left|x_{1}\right|^{-1}, k \in \mathbb{N}_{0}$.

Proof. Using the well-known $q$-binomial theorem (see [19], p. 241-248, [5], p. 416) and from (21), we get

$$
\begin{aligned}
& \sum_{n=0}^{\infty} g_{n}^{(s)}(\lambda, x ; q) t^{n} \\
& \quad=\sum_{n=0}^{\infty} \frac{\left(q^{s+1} ; q\right)_{n}}{(q ; q)_{n}}{ }_{2} \phi_{1}\left[\begin{array}{c}
q^{-n}, q^{\lambda} \\
q^{-s-n}
\end{array} ;, x\right] t^{n} \\
& \quad=\sum_{n=0}^{\infty} \frac{\left(q^{s+1} ; q\right)_{n}}{(q ; q)_{n}} \sum_{k=0}^{n} \frac{\left(q^{-n} ; q\right)_{k}\left(q^{\lambda} ; q\right)_{k}}{(q ; q)_{k}\left(q^{-s-n} ; q\right)_{k}} x^{k} t^{n} .
\end{aligned}
$$

Now making use of the identity

$$
\left(q^{-n} ; q\right)_{k}=\frac{(q ; q)_{n}}{(q ; q)_{n-k}}(-1)^{k} q^{\left(\begin{array}{c}
k \\
2
\end{array}\right)-n k}
$$

we have

$$
\begin{aligned}
\sum_{n=0}^{\infty} g_{n}^{(s)}(\lambda, x ; q) t^{n}=\sum_{n=0}^{\infty} \frac{\left(q^{s+1} ; q\right)_{n}}{(q ; q)_{n}} & \sum_{k=0}^{n} \frac{(q ; q)_{n}\left[(-1)^{k} q^{\left(\begin{array}{l}
k \\
2
\end{array}\right)-n k}\right](q ; q)_{s+n-k}\left(q^{\lambda} ; q\right)_{k}}{(q ; q)_{n-k}(q ; q)_{s+n}\left[(-1)^{k} q^{\left(\begin{array}{c}
k \\
2
\end{array}\right)^{n} t^{n}-s k}\right](q ; q)_{k}} \\
= & \sum_{n=0}^{\infty} \sum_{k=0}^{n} \frac{\left(q^{s+1} ; q\right)_{n}(q ; q)_{s+n-k}\left(q^{\lambda} ; q\right)_{k} q^{s k}}{(q ; q)_{n-k}(q ; q)_{s+n}(q ; q)_{k}} x^{k} t^{n} \\
= & \sum_{n=0}^{\infty} \sum_{k=0}^{\infty} \frac{\left(q^{s+1} ; q\right)_{n+k}(q ; q)_{s+n}\left(q^{\lambda} ; q\right)_{k} q^{s k}}{(q ; q)_{n}(q ; q)_{s+n+k}(q ; q)_{k}} x^{k} t^{n+k} \\
= & \sum_{n=0}^{\infty} \frac{\left(q^{s+1} ; q\right)_{n}}{(q ; q)_{n}} \sum_{k=0}^{\infty} \frac{\left(q^{\lambda} ; q\right)_{k} q^{s k}}{(q ; q)_{k}} x^{k} t^{n+k} \\
= & \sum_{n=0}^{\infty} \frac{\left(q^{s+1} ; q\right)_{n}}{(q ; q)_{n}} t^{n} \sum_{k=0}^{\infty} \frac{\left(q^{\lambda} ; q\right)_{k}}{(q ; q)_{k}}\left(q^{s} x t\right)^{k} \\
= & \frac{\left(1-q^{s+1} t\right)_{\infty}}{(1-t)_{\infty}} \frac{\left(1-q^{\lambda+s} x t\right)_{\infty}}{\left(1-q^{s} x t\right)_{\infty}} \\
& \frac{1}{(t ;)_{s+1}\left(q^{s} x t ; q\right)_{\lambda}},
\end{aligned}
$$

which completes the proof.

\section{The $q$-Generating Relations}

In this section, we have obtained bilinear and bilateral generating functions of various families for the $q$-analogue of the generalized Cesàro polynomials $g_{n, q}^{(s)}(\lambda, x)$ given by $(22)$. In addition, we will get a specific linear $q$-generating relationship that includes the basic analogue of Fox's $H$-function and a general class of $q$-hypergeometric polynomials. We begin by stating the following theorem.

Theorem 5. For nonvanishing function $\Omega_{\mu}\left(y_{1}, \ldots, y_{s}\right)$ of complex variables $y_{1}, \ldots, y_{s}(s \in \mathbb{N})$ and of complex order $\mu$, let

$$
\begin{array}{r}
\Lambda_{\mu, \psi}\left(y_{1}, \ldots, y_{s} ; \zeta\right):=\sum_{k=0}^{\infty} a_{k} \Omega_{\mu+\psi k}\left(y_{1}, \ldots, y_{s}\right) \zeta^{k}, \\
\left(a_{k} \neq 0, \mu, \psi \in \mathbb{C}\right)
\end{array}
$$

and

$$
\begin{aligned}
\Theta_{n, p}^{\mu, \psi}\left(\lambda, x ; q ; y_{1}, \ldots, y_{s} ; \xi\right) \\
\quad:=\sum_{k=0}^{[n / p]} a_{k} g_{n-p k}^{(s)}(\lambda, x ; q) \Omega_{\mu+\psi k}\left(y_{1}, \ldots, y_{s}\right) \xi^{k},
\end{aligned}
$$

where $[n / p]$ denotes the integer part of $n, p \in \mathbb{R}$. 
Then,

$$
\begin{aligned}
& \sum_{n=0}^{\infty} \Theta_{n, p}^{\mu, \psi}\left(\lambda, x ; q ; y_{1}, \ldots, y_{s} ; \frac{\eta}{t^{p}}\right) t^{n} \\
& \quad=\frac{1}{\left(q^{s} x t ; q\right)_{\lambda}(t ; q)_{s+1}} \Lambda_{\mu, \psi}\left(y_{1}, \ldots, y_{s} ; \eta\right) .
\end{aligned}
$$

Proof. Let $S$ denote the first member of the assertion (28) of Theorem 5. Taking $\xi \longrightarrow \eta / t^{p}$ and sum from $n=0$ to $\infty$ and also multiplying by $t^{n}$, we have

$$
\begin{aligned}
S & =\sum_{n=0}^{\infty} \Theta_{n, p}^{\mu, \psi}\left(\lambda, x ; q ; y_{1}, \ldots, y_{s} ; \frac{\eta}{t^{p}}\right) t^{n} \\
& =\sum_{n=0}^{\infty} \sum_{k=0}^{[n / p]} a_{k} g_{n-p k}^{(s)}(\lambda, x ; q) \Omega_{\mu+\psi k}\left(y_{1}, \ldots, y_{s}\right) \eta^{k} t^{n-p k} .
\end{aligned}
$$

Replacing $n$ by $n+p k$, we can write

$$
\begin{aligned}
S & =\sum_{n=0}^{\infty} \sum_{k=0}^{\infty} a_{k} g_{n}^{(s)}(\lambda, x ; q) \Omega_{\mu+\psi k}\left(y_{1}, \ldots, y_{s}\right) \eta^{k} t^{n} \\
& =\sum_{n=0}^{\infty} g_{n}^{(s)}(\lambda, x ; q) t^{n} \sum_{k=0}^{\infty} a_{k} \Omega_{\mu+\psi k}\left(y_{1}, \ldots, y_{s}\right) \eta^{k} \\
& =\frac{1}{\left(q^{s} x t ; q\right)_{\lambda}(t ; q)_{s+1}} \Lambda_{\mu, \psi}\left(y_{1}, \ldots, y_{s} ; \eta\right) .
\end{aligned}
$$

which completes the proof.

Theorem 6. Let $\left\{S_{n, q}\right\}_{n=0}^{\infty}$ be an arbitrary bounded sequence, let $M, N, P, Q$ be positive integers such that $0 \leq M \leq Q, 0 \leq$ $N \leq P$, let $h>0$, and let $m$ be an arbitrary positive integer. Then the following bilateral $q$-generating relation holds:

$$
\begin{aligned}
& \sum_{n=0}^{\infty} S_{n, q} g_{n}^{(s)}(\lambda, \rho x ; q) \\
& \cdot H_{P+1, Q}^{M, N+1}\left[\begin{array}{c}
(1-\mu-n, h),(a, \alpha) \\
y ; q \mid
\end{array}\right] t^{n} \\
& =\frac{1}{2 \pi i} \int_{C} \Phi(u ; \beta) \\
& \cdot \frac{\Gamma_{q}(\mu+h u)(1-q)^{\mu+h u-1}}{(q ; q)_{\infty}} \times \sum_{k=0}^{\infty} \sum_{n=0}^{\infty} S_{n+k, q} \\
& \cdot \frac{\left(q^{\mu+h u} ; q\right)_{k}\left(q^{\mu+k+h u} ; q\right)_{n}\left(q^{s+1} ; q\right)_{n}\left(q^{\lambda} ; q\right)_{k}}{(q ; q)_{n}(q ; q)_{k}}\left(q^{s} \rho x t\right)^{k} \\
& \cdot y^{u} t^{n} d_{q} u, \quad
\end{aligned}
$$

where $0<|q|<1$ and $\rho$ and $\mu$ are arbitrary numbers.
Proof. Denoting, for convenience, the left-hand side of (31) by $L$ and using the contour integral representation (16) for the $q$-analogue of Fox's $H$-function and the definition (21) for the q-generalized Cesàro polynomials, we get

$$
\begin{aligned}
L & =\frac{1}{2 \pi i} \sum_{n=0}^{\infty} S_{n, q}\left(\frac{\left(q^{s+1} ; q\right)_{n}}{(q ; q)_{n}}{ }_{2} \phi_{1}\left[\begin{array}{c}
q^{-n}, q^{\lambda} \\
q^{-s-n}
\end{array} q, \rho x\right]\right) \\
& \cdot\left\{\int_{C} \Phi(u ; q) G\left(q^{\mu+n+h u)} y^{u} d_{q} u\right\} t^{n} .\right.
\end{aligned}
$$

Changing the order of summations and integration, we obtain

$$
\begin{aligned}
L= & \frac{1}{2 \pi i} \int_{C} \Phi(u ; q) \sum_{n=0}^{\infty} \sum_{k=0}^{n} S_{n, q} G\left(q^{\mu+n+h u)} l\right. \\
& \cdot \frac{\left(q^{s+1} ; q\right)_{n}}{(q ; q)_{n}} \frac{\left(q^{-n} ; q\right)_{k}\left(q^{\lambda} ; q\right)_{k}}{\left(q^{-s-n} ; q\right)_{k}(q ; q)_{k}}(\rho x)^{k} y^{u} t^{n} d_{q} u,
\end{aligned}
$$

where $\Phi(u ; q)$ is given by (17). Using of the relation for q-gamma function, namely,

$$
G\left(q^{\alpha}\right)=\frac{\Gamma_{q}(\alpha)(1-q)^{\alpha-1}}{(q ; q)_{\infty}},
$$

we obtain

$$
\begin{aligned}
L= & \frac{1}{2 \pi i} \int_{C} \Phi(u ; q) \frac{\Gamma_{q}(\mu+h u)(1-q)^{\mu+h u-1}}{(q ; q)_{\infty}} \\
& \cdot \sum_{n=0}^{\infty} \sum_{k=0}^{n} S_{n, q}\left(q^{\mu+h u} ; q\right)_{n} \\
& \cdot \frac{\left(q^{s+1} ; q\right)_{n}}{(q ; q)_{n}} \frac{\left(q^{-n} ; q\right)_{k}\left(q^{\lambda} ; q\right)_{k}}{\left(q^{-s-n} ; q\right)_{k}(q ; q)_{k}}(\rho x)^{k} y^{u} t^{n} d_{q} u .
\end{aligned}
$$

By using identity (24), we have

$L$

$$
\begin{aligned}
& =\frac{1}{2 \pi i} \int_{C} \Phi(u ; q) \\
& \cdot \frac{\Gamma_{q}(\mu+h u)(1-q)^{\mu+h u-1}}{(q ; q)_{\infty}} \sum_{n=0}^{\infty} \sum_{k=0}^{n} S_{n, q} \\
& \cdot \frac{\left(q^{\mu+h u} ; q\right)_{n}\left(q^{s+1} ; q\right)_{n}\left(q^{\lambda} ; q\right)_{k}(q ; q)_{s+n-k}}{(q ; q)_{s+n}(q ; q)_{n-k}(q ; q)_{k}} q^{k s}(\rho x)^{k} \\
& \cdot y^{u} t^{n} d_{q} u .
\end{aligned}
$$

Again, changing the order of summations and making use of the series rearrangement relation [1]

$$
\sum_{n=0}^{\infty} \sum_{k=0}^{n} B(k, n)=\sum_{k=0}^{\infty} \sum_{n=0}^{\infty} B(k, n+k),
$$


we obtain

$L$

$$
\begin{aligned}
& =\frac{1}{2 \pi i} \int_{C} \Phi(u ; q) \\
& \cdot \frac{\Gamma_{q}(\mu+h u)(1-q)^{\mu+h u-1}}{(q ; q)_{\infty}} \sum_{k=0}^{\infty} \sum_{n=0}^{\infty} S_{n+k, q} \\
& \cdot \frac{\left(q^{\mu+h u} ; q\right)_{n+k}\left(q^{s+1} ; q\right)_{n+k}\left(q^{\lambda} ; q\right)_{k}(q ; q)_{s+n}}{(q ; q)_{s+n+k}(q ; q)_{n}(q ; q)_{k}} q^{k s}(\rho x)^{k} \\
& \cdot y^{u} t^{n+k} d_{q} u .
\end{aligned}
$$

Now by interchanging the order of contour integral and summation, and using the q-identities [4], namely,

$$
(\alpha ; q)_{n+k}=(\alpha ; q)_{n}(\alpha q ; q)_{k}
$$

and

$$
(\alpha ; q)_{n}=\frac{\Gamma(\alpha+n)(1-q)^{n}}{\Gamma(\alpha)} \quad(n>0),
$$

we obtain

$L$

$$
\begin{aligned}
& =\frac{1}{2 \pi i} \int_{C} \Phi(u ; q) \\
& \cdot \frac{\Gamma_{q}(\mu+h u)(1-q)^{\mu+h u-1}}{(q ; q)_{\infty}} \sum_{k=0}^{\infty} \sum_{n=0}^{\infty} S_{n+k, q} \\
& \cdot \frac{\left(q^{\mu+h u} ; q\right)_{k}\left(q^{\mu+k+h u} ; q\right)_{n}\left(q^{s+1} ; q\right)_{n}\left(q^{\lambda} ; q\right)_{k}}{(q ; q)_{n}(q ; q)_{k}}\left(q^{s} \rho x t\right)^{k} \\
& \cdot y^{u} t^{n} d_{q} u .
\end{aligned}
$$

\section{Special Cases}

As an application of the above in Theorem 5, when the multivariable function $\Omega_{\mu+\psi k}\left(y_{1}, \ldots, y_{r}\right), k \in \mathbb{N}_{0}, r \in \mathbb{N}$, is expressed in terms of simpler functions of one and more variables, then we can give additional applications of the above theorem.

We first set

$$
\begin{aligned}
r & =1 \\
\text { and } \Omega_{\mu+\psi k}(y) & =g_{\mu+\psi k}^{(s)}(\lambda, y ; q)
\end{aligned}
$$

in Theorem 5, where the q-generalized Cesàro polynomials are generated by (22). We thus led to the following result which provides a class of bilinear generating functions for the q-generalized Cesàro polynomials.
Corollary 1. If

$$
\begin{aligned}
& \Lambda_{\mu, \psi}(\lambda, y ; q ; \zeta):=\sum_{k=0}^{\infty} a_{k} g_{\mu+\psi k}^{(s)}(\lambda, y ; q) \zeta^{k}, \\
& a_{k} \neq 0, \mu, \psi \in \mathbb{C},
\end{aligned}
$$

then we have

$$
\begin{gathered}
\sum_{n=0}^{\infty} \sum_{k=0}^{[n / p]} a_{k} g_{n-p k}^{(s)}(\lambda, x ; q) g_{\mu+\psi k}^{(s)}(\lambda, y ; q) \frac{\eta^{k}}{t^{p k}} t^{n} \\
=\frac{1}{\left(q^{s} x t ; q\right)_{\lambda}(t ; q)_{s+1}} \Lambda_{\mu, \psi}(\lambda, y ; q ; \eta) .
\end{gathered}
$$

Remark 2. Using the generating relation (22) for the generalized Cesàro polynomials and getting $a_{k}=1, \mu=0, \psi=1$ in Corollary 1, we find that

$$
\begin{gathered}
\sum_{n=0}^{\infty} \sum_{k=0}^{[n / p]} g_{n-p k}^{(s)}(\lambda, x ; q) g_{k}^{(s)}(\lambda, y ; q) \eta^{k} t^{n-p k} \\
=\frac{1}{\left(q^{s} x t ; q\right)_{\lambda}(t ; q)_{s+1}} \frac{1}{\left(q^{s} y \eta ; q\right)_{\lambda}(\eta ; q)_{s+1}}
\end{gathered}
$$

where $|t|<1,|\eta|<1$.

By assigning suitable special values to the sequence $\left\{S_{n, q}\right\}_{n=0}^{\infty}$, our main result (Theorem 6) can be applied to derive certain bilateral $q$-generating relations for the product of orthogonal $q$-polynomials and the basic analogue of Fox's $H$-function. To illustrate this, we consider the following example.

Set $\rho=1$ and

$$
S_{n, q}=\frac{1}{\left(q^{\lambda} ; q\right)_{k}\left(q^{s+1} ; q\right)_{n-k}} .
$$

Thus, in view of the above relations, Theorem 6 yields the $q$-generating relation involving $q$-generalized Cesàro polynomial and the basic Fox's $H$-function as below.

Corollary 3. The following bilateral generating function holds true:

$$
\begin{aligned}
& \sum_{n=0}^{\infty} \frac{1}{\left(q^{\lambda} ; q\right)_{k}\left(q^{s+1} ; q\right)_{n-k}} g_{n}^{(s)}(\lambda, \rho x ; q) \\
& \cdot H_{P+1, Q}^{M, N+1}\left[\begin{array}{cc}
(1-\mu-n, h),(a, \alpha) \\
y ; q \mid & (b, \beta)
\end{array}\right] t^{n}=\frac{1}{(1-t)^{(\mu)}}
\end{aligned}
$$




$$
\begin{aligned}
& \cdot \sum_{k=0}^{\infty} \frac{\left(q^{s} x t\right)^{k}}{\left(t q^{\mu} ; q\right)_{k}(q ; q)_{k}} \frac{1}{2 \pi i} \\
& \cdot \int_{C} \Phi(u ; q) \frac{\Gamma_{q}(\mu+k+h u)(1-q)^{\mu+k+h u-1}}{\left(t q^{\mu+k} ; q\right)_{h u}(q ; q)_{\infty}} y^{u} d_{q} u \\
& =\frac{1}{(1-t)^{(\mu)}} \sum_{k=0}^{\infty} \frac{\left(q^{s} x t\right)^{k}}{\left(t q^{\mu} ; q\right)_{k}(q ; q)_{k}} \\
& \cdot H_{P+1, Q}^{M, N+1}\left[\frac{y}{\left(1-t q^{\mu+k}\right)^{(h)}} ; q \mid \begin{array}{c}
(1-\mu-k, h),(a, \alpha) \\
(b, \beta)
\end{array}\right],
\end{aligned}
$$

where $|t|<1,0<|q|<1$ and $\mu$ is arbitrary numbers.

If we take $\alpha_{i}=\beta_{j}=1$ for all $i$ and $j$ and $m=h=1$ and set (19) and

$$
S_{n, q}=\frac{1}{\left(q^{\lambda} ; q\right)_{k}\left(q^{s+1} ; q\right)_{n-k}},
$$

in Theorem 6, we have the following bilateral generating functions for the $q$-generalized Cesàro polynomials.

Corollary 4. Let $\left\{S_{n, q}\right\}_{n=0}^{\infty}$ be an arbitrary bounded sequence and let $M, N, P, Q$ be positive integers satisfying $0 \leq M \leq$ $Q, 0 \leq N \leq P$. Then the following bilateral q-generating relation for the function $G_{q}(\cdot)$ holds:

$$
\begin{aligned}
& \sum_{n=0}^{\infty} \frac{1}{\left(q^{\lambda} ; q\right)_{k}\left(q^{s+1} ; q\right)_{n-k}} g_{n}^{(s)}(\lambda, \rho x ; q) \\
& \cdot G_{P+1, \mathrm{Q}}^{M, N+1}\left[\begin{array}{c}
1-\mu-n, a_{1}, \ldots, a_{P} \\
y ; q \mid \\
b_{1}, \ldots, b_{\mathrm{Q}}
\end{array}\right] t^{n} \\
& \quad \frac{1}{(1-t)^{(\mu)}} \sum_{k=0}^{\infty} \frac{\left(q^{s} \rho x t\right)^{k}}{\left(t q^{\mu} ; q\right)_{k}(q ; q)_{k}} \\
& \cdot G_{P+1, \mathrm{Q}}^{M, N+1}\left[\frac{y}{\left(1-t q^{\mu+k}\right)^{(h)}} ; q \mid \begin{array}{c}
1-\mu-k, a_{1}, \ldots, a_{P} \\
b_{1}, \ldots, b_{\mathrm{Q}}
\end{array}\right]
\end{aligned}
$$

where $|t|<1,0<|q|<1$ and $\rho$ and $\mu$ are arbitrary numbers.

For every suitable choice of the coefficients $a_{k}\left(k \in \mathbb{N}_{0}\right)$, if the multivariable function $\Omega_{\mu}\left(y_{1}, \ldots, y_{s}\right)(s=2,3, \ldots)$ is expressed as an appropriate product of several simpler functions, the assertion of the above Theorem 5 can be applied in order to derive various families of multilinear and multilateral generating functions for the $q$-generalized Cesàro polynomials $g_{n}^{(s)}(\lambda, x ; q)$ defined by (22).

We conclude with the remark that by suitably assigning values to the sequence $\left\{S_{n, q}\right\}_{n=0}^{\infty}$, the $q$-generating relation (31), being of general nature, will lead to several generating relations for the product of orthogonal $q$-polynomials and the basic analogue of the Fox's $H$ - functions.

\section{Data Availability}

No data were used to support this study.

\section{Conflicts of Interest}

The author declares that he has no conflicts of interest.

\section{References}

[1] H. M. Srivastava and H. L. Manocha, in A Treatise on Generating Functions, Halsted Press (Ellis Horwood Limited, Chichester), John Wiley and Sons, New York, NY, USA, 1984.

[2] S.-D. Lin, S.-J. Liu, H.-C. Lu, and H. M. Srivastava, "Integral representations for the generalized Bedient polynomials and the generalized Cesàro polynomials," Applied Mathematics and Computation, vol. 218, no. 4, pp. 1330-1341, 2011.

[3] N. Özmen and E. Erkus-Duman, "Some families of generating functions for the generalized Cesáro polynomials," Journal of Computational Analysis and Applications, vol. 25, no. 4, pp. 670683, 2018.

[4] G. Gasper and M. Rahman, Basic Hypergeometric Series, Cambridge University Press, Cambridge, UK, 2004.

[5] V. K. Jain and H. M. Srivastava, "Some families of multilinear q-generating functions and combinatorial q-series identities," Journal of Mathematical Analysis and Applications, vol. 192, no. 2, pp. 413-438, 1995.

[6] M. Asif, On Some Problems of Special Functions, Department of Applied Mathematics, Aligarh Muslim University, 2010, http://hdl.handle.net/10603/55628.

[7] H. S. Cohl, R. S. Costas-Santos, and T. V. Wakhare, "Some generating functions for q-polynomials," New Zealand Journal of Mathematics, vol. 10, no. 12, p. 758, 2018.

[8] R. K. Saxena, G. C. Modi, and S. L. Kalla, "A basic analogue of Fox's H-function," Revista Tecnica de la Facultad de Ingenieria Universidad del Zulia, vol. 6, no. Special Issue, pp. 139-143, 1983.

[9] A. M. Mathai and R. K. Saxena, Generalized Hypergeometric Functions with Applications in Statistics and Physical Sciences, Lecture Series in Mathematics, vol. 348, Springer-Verlag, New York, NY, USA, 1973.

[10] A. M. Mathai and R. K. Saxena, The H-Function with Applications in Statistics and Other Disciplines, Halsted Press [John Willey and Sons], New York-London-Sidney, 1978.

[11] H. M. Srivastava, K. C. Gupta, and S. P. Goyal, The H-Functions of One and Two Variables with Applications, South Asian Publications, New Delhi, 1982.

[12] A. M. Mathai, R. K. Saxena, and H. J. Haubold, The H-function: Theory and Applications, Springer, New York, NY, USA, 2010.

[13] R. K. Yadav and S. D. Purohit, "On application of Weyl fractional q-integral operator to generalized basic hypergeometric functions," Kyungpook Mathematical Journal, vol. 46, no. 2, pp. 235-245, 2006.

[14] R. K. Yadav, S. D. Purohit, and S. L. Kalla, "On generalized Weyl fractional q-integral operator involving generalized basic hypergeometric functions," Fractional Calculus \& Applied Analysis. An International Journal for Theory and Applications, vol. 11, no. 2, pp. 129-142, 2008.

[15] M. Mursaleen and M. Nasiruzzaman, "Approximation of modified jakimovski-leviatan-beta type operators," Constructive Mathematical Analysis, vol. 1, no. 2, pp. 88-98, 2018. 
[16] R. Maurya, H. Sharma, and C. Gupta, "Approximation properties of kantorovich type modifications of (p,q)-meyer-königzeller operators," Constructive Mathematical Analysis, vol. 1, no. 1, pp. 58-72, 2018.

[17] A. Altn, E. Erkus, and F. Tasdelen, "The q-Lagrange polynomials in several variables," Taiwanese Journal of Mathematics, vol. 10, no. 5, pp. 1131-1137, 2006.

[18] E. Erkus-Duman, "A q-extension of the Erkus-Srivastava polynomials in several variables," Taiwanese Journal of Mathematics, vol. 12, no. 2, pp. 539-543, 2008.

[19] L. J. Slater, Generalized Hypergeometric Functions, Cambridge University Press, Cambridge, London, UK, New York, NY, USA, 1966. 


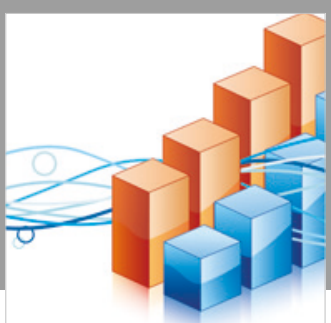

Advances in

Operations Research

\section{-n-m}
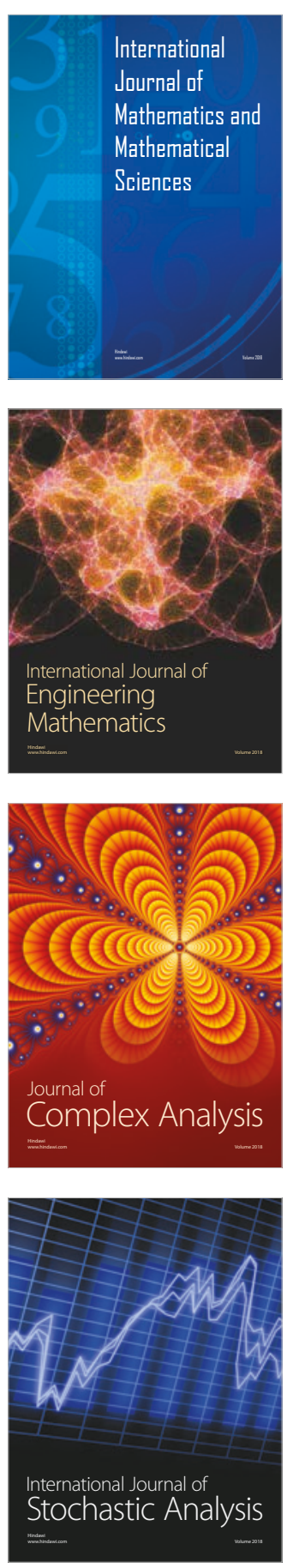
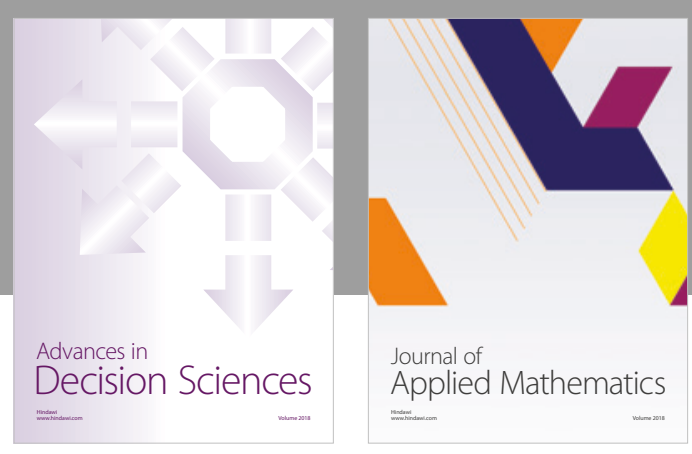

Journal of

Applied Mathematics
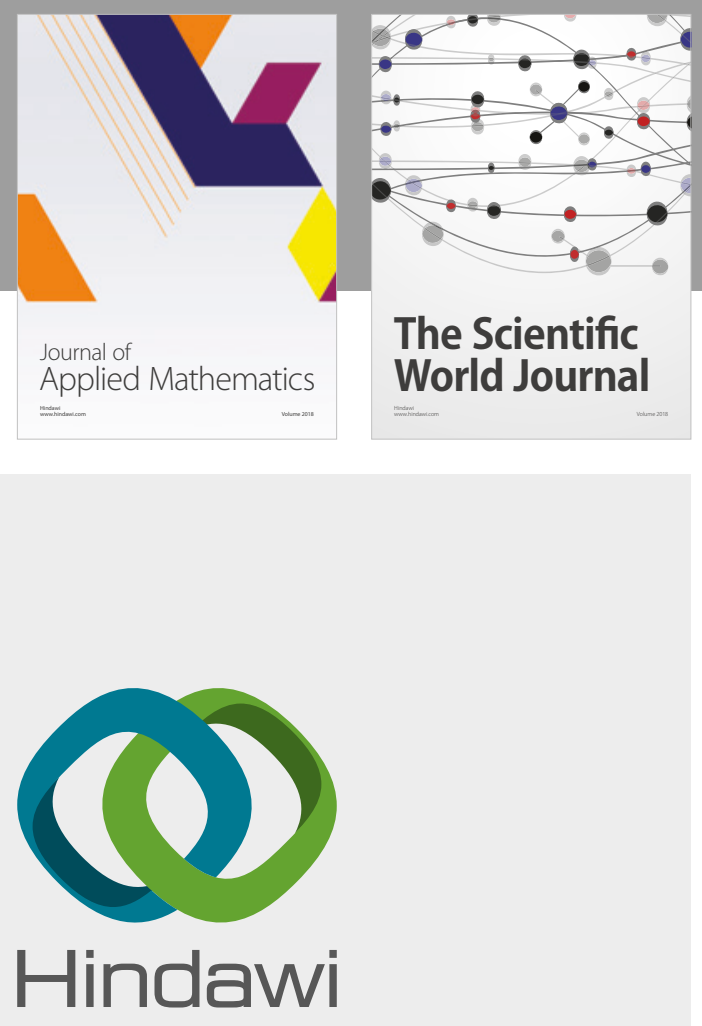

Submit your manuscripts at

www.hindawi.com

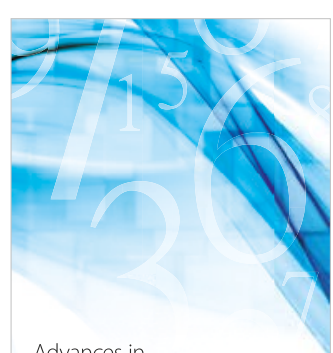

Advances in
Numerical Analysis
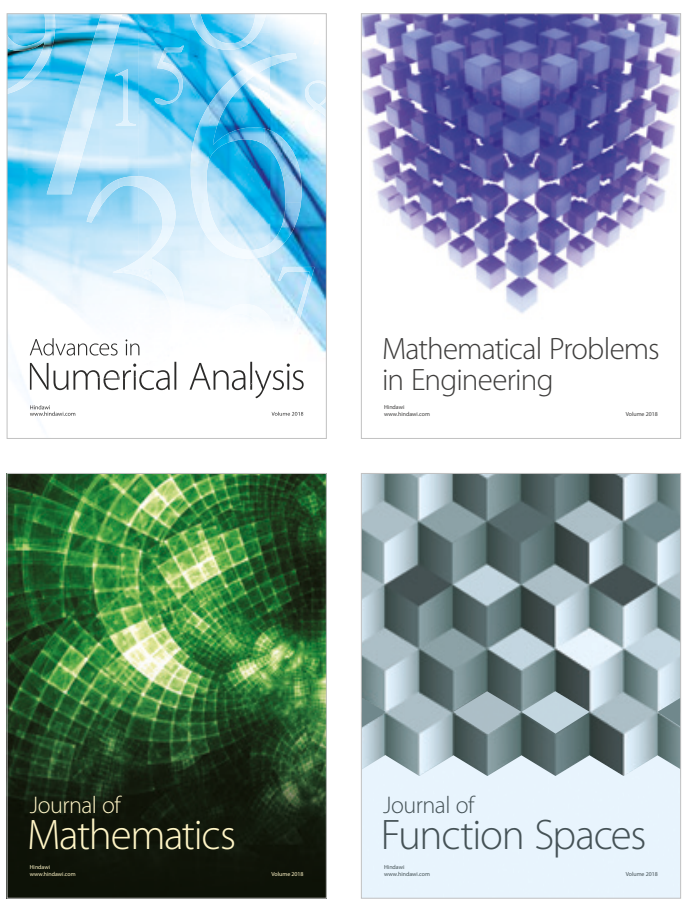

Mathematical Problems in Engineering

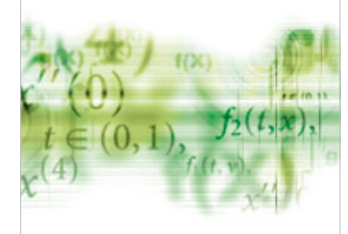

International Journal of

Differential Equations

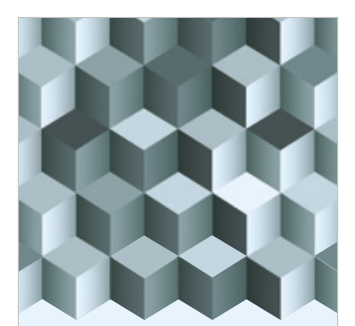

Journal of

Function Spaces

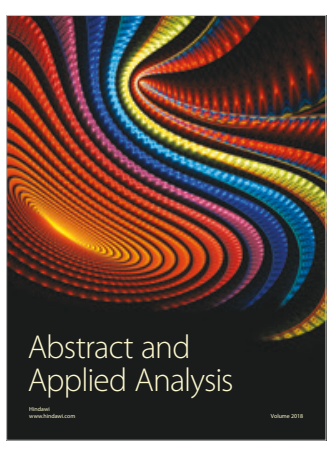

The Scientific

World Journal

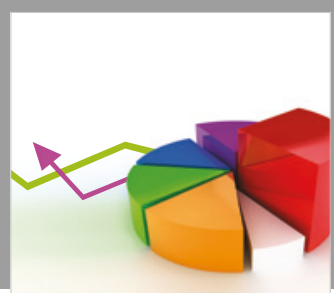

Journal of

Probability and Statistics
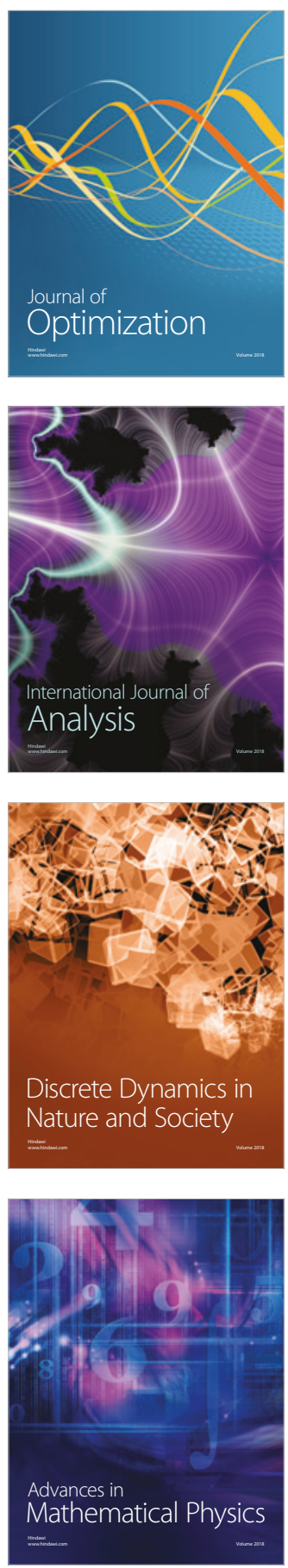